\title{
Testing Stage-Specific Effects of a Stage-Matched Intervention: A Randomized Controlled Trial Targeting Physical Exercise and Its Predictors
}

\author{
Sonia Lippke, $\mathrm{PhD}$ \\ Ralf Schwarzer, PhD \\ Jochen P. Ziegelmann, PhD \\ Urte Scholz, PhD \\ Benjamin Schüz, PhD
}

\begin{abstract}
Health education interventions can be tailored toward stages of change. This strategy is based on theories that predict at which stage which variables are indicative of subsequent behavior change processes. For example, planning is regarded as being effective in intenders. However, rather few studies have tested whether matched interventions are more successful for stage transitions than mismatched ones. Also very few previous studies have identified specific variables as targets of stage-matched interventions. A 2 (condition) $\times 2$ (stages) experimental study tested the effects of stage-matched interventions for 226 participants. The stagematched intervention moved significantly more individuals forward to action than did the control condition. Stage-specific effects were found to corroborate $78 \%$ of the assumptions. Multiple mediator analyses revealed stage-specific mechanisms, indicating that intention and planning facilitated behavior change in intenders. Thus, health behavior interventions should take stages of change into account.
\end{abstract}

Keywords: physical activity; intention; planning; self-efficacy; stage; multiple mediator analyses

Helping individuals to change their behavior is most promising if interventions take into account characteristics of the individual. Previous behavior and motivation are crucial characteristics differentiating among individuals and thus should guide intervention development. This idea is reflected in stage models of health behavior, which assume that individuals progress through an ordered set of stages while contemplating, initiating, and maintaining health behavior change (Weinstein, Rothman, \& Sutton, 1998). The main dependent variable in stage theories is stage progression, instead of behavior change. Stage progression can obviously occur, for example, when individuals move from deciding to change to actual behavior change but also when individuals move from being undecided to deciding to adopt a behavior. This implies that such health promotion programs should be evaluated regarding stage progression instead of dichotomous

Sonia Lippke, Ralf Schwarzer, and Jochen P. Ziegelmann, Freie Universität Berlin, Germany. Urte Scholz, Universität Zürich, Switzerland. Benjamin Schüz, German Centre of Gerontology, Berlin, Germany.

Address correspondence to Sonia Lippke, Freie Universität Berlin, Health Psychology, Habelschwerdter Allee 45 (PF10), 14195 Berlin, Germany; phone: (+49) 30-838-55619; e-mail: s.lippke@fu-berlin.de.

Health Education \& Behavior, Vol. 37(4): 533-546 (August 2010)

DOI: $10.1177 / 1090198109359386$

(C) 2010 by SOPHE 
behavior change, a criterion mismatched to individuals who have not yet decided to change their behavior at all (Weinstein, Rothman, \& Sutton, 1998). Stage theories suggest factors that should be targeted in specific stage groups to achieve maximum intervention effectiveness (e.g., Weinstein, Lyon, Sandman, \& Cuite, 1998). However, there is still a relative lack of studies examining the superiority of such stage-matched interventions for health promotion. The current study therefore tests at which stage group a planning intervention is more effective. In addition, we also aim to explain which variables produce the effect of the intervention on behavior.

The theoretical backdrop of the study is the health action process approach (HAPA; Schwarzer et al., 2007; Schwarzer, Luszczynska, Ziegelmann, Scholz, \& Lippke, 2008). The HAPA has been selected as the framework for the present study because of two main reasons: (a) the HAPA explicitly predicts which factors will be effective at which stages (see below) and (b) there is ample evidence for stage-specific effects of these factors, which supports the HAPA stage assumptions (e.g., Lippke, Ziegelmann, \& Schwarzer, 2004).

\section{Stages and Mechanisms}

The HAPA makes a distinction between different stages (Schwarzer et al., 2007). The basic idea is that individuals experience psychological changes when moving from the first stage (nonintentional) to the second (intentional). Intenders are individuals not yet acting according to their intentions in contrast to individuals acting according to their intentions (actors). The HAPA predicts that the higher the levels of risk awareness and positive outcome expectancies, the more likely a nonintender is to form a behavioral intention. As soon as the intention is high enough, a nonintender becomes an intender. Intenders are in need of preparing and planning the behavioral change to become actors. Thus, providing individuals in different stages with stage-specific support in these factors might advance movement toward the goal behavior. The HAPA specifies stagespecific factors predicting stage progression.

After a person has formed the intention to perform a new behavior (and has thereby entered the volitional stage), this intention has to be maintained and translated into detailed instructions on how to perform the desired action by planning (Gollwitzer \& Sheeran, 2006; Ziegelmann, Lippke, \& Schwarzer, 2006). Planning involves linking situation parameters (when, where) to a predefined sequence of action (how). Action planning refers to plans regarding the initiation of health behaviors, whereas the term coping planning refers to dealing with problems regarding the maintenance of health behaviors (Ziegelmann et al., 2006). Both are important components of volitional planning interventions. However, it has been suggested that such plans are subordinate to intentions and unfold their efficacy particularly when intentions for the respective behavior are high (Gollwitzer \& Sheeran, 2006).

Volitional self-efficacy describes optimistic self-beliefs concerning the ability to cope with the experience of possible failure and recovery from setbacks. It is thus most important when it comes to resuming an interrupted behavioral chain in direction of the behavioral intention. This stage-specific self-efficacy belief has been examined in several domains of behavior change (Scholz, Sniehotta, \& Schwarzer, 2005; Schwarzer et al., 2007; Schwarzer et al., 2008). For example, Scholz and colleagues (2005) found volitional self-efficacy to be most effective in terms of behavior change for individuals who had indeed experienced a lapse back to physical inactivity and not for those who maintained their physical activity regimen over time. 


\section{Experimental Evidence for Stage-Matched Interventions}

The strongest empirical support for the assumptions made by stage theories stems from experimental matched-mismatched research designs (Weinstein et al., 1998). This describes a factorial design in which individuals in different stages are randomized toward receiving either a stage-matched or a stage-mismatched intervention. In terms of the HAPA stages, a motivational intervention (intention formation) would be matched to individuals in the nonintentional stage and a mismatched intervention to those in the volition stage. A volitional intervention focusing on volitional processes (e.g., planning) would accordingly be matched to intenders and mismatched to nonintenders. In an experimental study with patients partaking in an orthopedic rehabilitation, such stage-specific effects of a volitional treatment (i.e., a planning intervention) were demonstrated (Lippke et al., 2004). The volitional intervention did help intenders translate their intentions into action. Nonintenders, however, did not benefit from the treatment in such ways. However, no studies could be found on how (i.e., via which processes or mediators) the stage-matched interventions work.

\section{Aims of the Study}

The study aims at examining research questions on two levels - an outcome level, where we examine differential results according to experimental condition, and a process level, where we examine whether the intervention changes mediators and whether these mediators are responsible for stage transitions and behavior changes. At the outcome level, the study aims at testing (Hypothesis 1) whether stage transitions are more likely in individuals receiving a treatment matched to their stage than those in the control condition or individuals in a stage-mismatched condition. At the process level, the study tests whether the intervention changed stage-specific factors. In particular, it was hypothesized that a volitional intervention should not change risk awareness (Hypothesis 2a) or outcome expectancies (Hypothesis $2 \mathrm{~b}$ ) neither in nonintenders nor intenders (as risk awareness and outcome expectancies are seen as motivational variables). Furthermore, (Hypothesis 2c) it is assumed that intention is not changed by the volitional treatment in comparison to the control condition in all individuals (as this is seen as a motivational variable). (Hypothesis $2 \mathrm{~d}$ ) Volitional self-efficacy should be affected by the volitional intervention only in intenders (matched condition). (Hypothesis 2e) The same pattern is expected for planning. Finally, (Hypothesis 2f) actual behavior change is expected only for intenders receiving the volitional treatment. These hypotheses result in 18 testable predictions (see Table 3). Also at the process level, we test stagespecific mediation mechanisms, that is, how the intervention translates into behavior change. It is hypothesized, that the effect of the volitional intervention is mediated by changes in (Hypothesis 3a) intention, (Hypothesis 3b) planning, and (Hypothesis 3c) volitional self-efficacy in intenders. (Hypothesis 3d) No such mediation mechanisms are expected in nonintenders.

\section{METHOD}

A Web-based randomized controlled trial was performed using the software dynQuest (Rademacher \& Lippke, 2007). The study applied a volitional treatment and a control condition and included two measurement points in time, 5 weeks apart. 


\section{Participants}

A total of 1,279 potential study participants visited the start page of the Web site. Of these, 881 people $(68.9 \%)$ provided their e-mail address to receive an invitation for a follow-up assessment. In all, 365 respondents $(41.4 \%$ of those who could potentially participate) answered the follow-up questionnaire. Dropout analyses in terms of sex, Time 1 (T1) behavior, partner status, and college education showed no significant differences between the initial sample and those who completed both measurement points in time. Significant differences $(p<.05)$ between dropouts and study participants appeared in terms of age (dropouts were on average 2 years younger). Similarly, no differences were found in $\mathrm{T} 1$ social-cognitive variables. Thus, the longitudinal sample can be considered roughly representative of the initial one.

Participants were included in the analyses only if they did not meet goal behavior prior to the first measurement point and if they completed the assessments at both points in time (as the volitional planning intervention should be matched to the intenders only; Lippke et al., 2004). Thus, the final longitudinal sample consisted of 226 participants, aged 18 to $64(M=37.16, S D=9.74)$; of these, $82.3 \%$ were women. About $59.7 \%$ of respondents were living with a partner, $71.7 \%$ had completed senior high school, and $44.2 \%$ held a university degree. The sample is comparable to those of other online studies (see Wiedemann et al., 2009), and the results of the dropout analysis suggest that the longitudinal sample is roughly representative of the baseline sample.

\section{Procedure}

Respondents were recruited at T1 by means of invitations sent via e-mail and advertisements placed on a university Web site with a link to the questionnaire. Such a recruitment strategy that implicates self-selection of participants is commonly used in health behavior change studies (see Prochaska, Wright, \& Velicer, 2008).

After the study was introduced, participants provided informed consent and followed a link to a self-administered questionnaire, followed by a random assignment to either the volitional intervention or the control condition. Five weeks later, at Time 2 (T2), all participants who provided their e-mail address were invited by e-mail to answer a followup questionnaire.

\section{Measures}

We assessed the following variables in our study. The response format for all items was a 4-point Likert-type scale ranging from 1 (disagree) to 4 (agree), if not otherwise reported in the following. Risk awareness was measured by three items assessing situation outcome expectations. The stem "If I keep my lifestyle the way it is . .." was followed by the items "there is a high likelihood that I will develop severe health problems," "then I will develop a severe disease (such as heart attack)," and "then I will gain weight" (Cronbach's $\alpha=.80$ and .84 for T1 and T2, respectively). The scale was validated in a previous study (Schwarzer et al., 2007) with regard to its predictive power for intentions.

Outcome expectancies regarding behavior change were assessed with four items (Cronbach's $\alpha=.65$ and .62 for T1 and T2, respectively). All items had the stem "If I will exercise on a regular basis ..." followed by positive consequences "then I will be more resilient for everyday life," "then it will be good for my health," "then I will just 
feel better afterwards," and "then I will look better." The scale was validated in previous studies (Lippke, Ziegelmann, Schwarzer, \& Velicer, 2009; Schwarzer et al., 2007) with regard to its ability to predict intentions to change behavior.

Intentions were measured by four items (Cronbach's $\alpha=.81$ and .86 for T1 and T2, respectively). The stem "I intend to ..." was followed by the items "be physically active on a weekly basis," "be regularly physically active so that I will sweat," "be physically active on a regular basis (to run, swim, cycle etc.)," and "be physically active at least three times per week for 30 minutes so that I will sweat." The scale was validated in previous studies (Schwarzer et al., 2007) for various health behaviors.

Volitional self-efficacy was assessed by three items (Cronbach's $\alpha=.87$ and .90 for $\mathrm{T} 1$ and T2, respectively) with the stem "I am confident that I can resume a physically active lifestyle. ..." The items then were "even if I have postponed my concrete plans for a couple of times," "even if I have not exercised for some time," and "even if I have not exercised for some weeks." The validity of the scale in terms of stage specificity was established in previous studies (Scholz, Schüz, Ziegelmann, Lippke, \& Schwarzer, 2008; Schwarzer et al., 2007).

Planning was assessed by four items (Cronbach's $\alpha=.85$ at both measurement points). The item stem "I have made a detailed plan regarding ..." was followed by the items "when to exercise," "where to exercise," "how to exercise," and "how often to exercise." These assessments of planning have proved valid predictors of subsequent behavior changes (Lippke et al., 2009; Scholz et al., 2008).

Physical exercise at T1 and T2 was assessed by using one item of the International Physical Activity Questionnaire (IPAQ; Craig et al., 2003). Participants were asked to indicate how often during the past 7 days they had engaged in vigorous physical activities such as running, swimming, and cycling. Moreover, they were asked how much time they had usually spent performing these activities on each of these days. Frequency and average duration per session were then multiplied to obtain a measure of weighted duration of vigorous physical exercise during the past 7 days. In addition, study participants were asked, "During the past week, did you perform physical exercises additionally to your daily activities and work chores?" Answers were given on a rating scale including the options 1 (no), 2 (yes, sometimes; at least once per month), 3 (yes, regularly; at least once per week), 4 (yes, regularly at least twice per week for 20 minutes or more), and 5 (yes, regularly at least three times per week for 30 minutes or more). This was done to validate the data measured with the IPAQ and to assess physical activity with a higher scale correspondence toward the social-cognitive predictors of behavior (correlation of the two assessments: T1 $r=.56, p<.01$; T2 $r=.58, p<.01$ ). The IPAQ was formerly validated with acceptable psychometric properties in 12 countries (Craig et al., 2003).

Stage was assessed asking study participants, "Are you physically active on a weekly basis at least for 30 minutes so that you sweat and breathe hard?" Those indicating yes at T1 were diagnosed as meeting the goal behavior and were excluded from the study as those individuals were not in need of interventions to help them increase their activity level. Those answering no were classified as nonintenders if they did not fully agree with the intention items (below or equal to $3 ; n=92,40.7 \%$ ). Those replying no and who agreed very highly with the intention items (above $3.01 ; n=134,59.3 \%$ ) were categorized as intenders (Lippke et al., 2009). At T2, the same assessment was used: Study participants answering that they were not physically active were diagnosed as nonintenders (below or equally 3 on the intention index) or intenders (above 3.01). Those indicating being active were categorized as actors (and not excluded from the analyses). The stage algorithm was validated against other measures of behavioral stages in a previous study (Lippke et al., 2009). 


\section{Intervention}

The software dynQuest (Rademacher \& Lippke, 2007) randomized all individuals to one of the two groups: (a) volitional intervention or (b) control condition. The volitional planning intervention was based on previous interventions such as the one by Lippke et al. (2004) and Ziegelmann et al. (2006) and consisted of a page on which participants were encouraged to write down up to five physical activities that they intended to perform. Then they were encouraged to form action plans such as "I will perform the following physical activities . . ." by specifying when, where, and how (Gollwitzer \& Sheeran, 2006). In addition, barriers to be anticipated and plans to overcome these difficulties were formed. With the instruction, "What could keep you from exercising? How could you be physically active in spite of these obstacles?" coping planning was aimed at (Ziegelmann et al., 2006). All questions were open ended. The control group did not receive a treatment but was directly linked to the last Web page of the questionnaire.

A randomization check revealed differences in gender (control group $90.4 \%$ female participants, intervention group $78.2 \% ; \chi^{2}=5.43, p=.02$ ). No differences between the control and treatment group were found regarding partner status, schooling and college or university degree, age, social-cognitive factors, stage, and behavior at T1 (all $p \mathrm{~s}>.16$ ).

\section{Analytical Procedure}

All analyses were run with IBM SPSS 15.0. Hypothesis 1 was tested with a $\chi^{2}$ test, correlation analyses for ordinal variables (Spearman's rho), and post hoc comparison for the two correlations. Hypotheses $2 \mathrm{a}$ to $2 \mathrm{f}$ were tested with ANCOVAs and post hoc tests comparing group means. Hypotheses on the multiple mediator models were performed using an SPSS macro (Preacher \& Hayes, 2008). This procedure was favored over the commonly used simple mediation analysis (e.g., Sobel-Goodman test) mainly because (a) running simple mediation analyses for every potential mediator individually increases the probability of Type I errors, (b) the multiple mediator models allow to use bootstrapping, a nonparametric resampling procedure, which does not impose theoften violated - assumption of normality of the sampling distribution (see Preacher \& Hayes, 2008), and (c) multiple mediator analysis allows testing of the significance of indirect effects directly. Residualized change scores were used for all variables in the multiple mediation analysis. Bootstrapping was applied with confidence intervals (CIs) generated from 5,000 resamples $\left(\mathrm{CI}_{\mathrm{BCA}}=\right.$ bias-corrected and accelerated CIs with $\alpha=.05$; MacKinnon, Lockwood, \& Williams, 2004). Missing data within each measurement point in time were imputed using the expectation maximization algorithm in SPSS (Enders, 2001).

\section{RESULTS}

\section{Stage Transitions (Testing Hypothesis 1)}

Differences in stage transitions according to the intervention were found only in intenders (see Table 1). The correlations of stage movements were $r=-.05(p=.33)$ for nonintenders and $r=.22(p<.01)$ and intenders. These two correlations were significantly different $(z=1.99, p=.01)$.

To compare stage-matched and stage-mismatched effects, the frequencies of stage transitions were compared: Participants randomized to the intervention group or control 
Table 1. Stage Transitions by Experimental Condition: Frequencies and Percentages

\begin{tabular}{|c|c|c|c|c|c|c|c|c|c|}
\hline \multirow{3}{*}{$\begin{array}{l}\text { Baseline } \\
\text { Stage }\end{array}$} & & \multicolumn{6}{|c|}{ Transition to Time 2} & & \\
\hline & & \multicolumn{2}{|c|}{ Nonintenders } & \multicolumn{2}{|c|}{ Intenders } & \multicolumn{2}{|c|}{ Actors } & \multicolumn{2}{|c|}{ Total } \\
\hline & & Freq. & $\%$ & Freq. & $\%$ & Freq. & $\%$ & Freq. & $\%$ \\
\hline \multirow[t]{2}{*}{$\begin{array}{l}\text { Nonintenders } \\
\text { (T1) }\end{array}$} & $\begin{array}{l}\text { Control } \\
\text { group }\end{array}$ & 19 & 55.9 & $\rightarrow 8$ & 23.5 & $\rightarrow 7$ & 20.6 & 34 & 100 \\
\hline & $\begin{array}{l}\text { Intervention } \\
\text { group }\end{array}$ & 35 & 60.3 & $\rightarrow 13$ & 22.4 & $\rightarrow 10$ & 17.2 & 58 & 100 \\
\hline \multirow[t]{2}{*}{$\begin{array}{l}\text { Intenders } \\
\text { (T1) }\end{array}$} & $\begin{array}{l}\text { Control } \\
\text { group }\end{array}$ & 16 & $32.0 \leftarrow$ & 21 & 42.0 & $\rightarrow 13$ & 26.0 & 50 & 100 \\
\hline & $\begin{array}{l}\text { Intervention } \\
\text { group }\end{array}$ & 15 & $17.9 \leftarrow$ & 30 & 35.7 & $\rightarrow 39$ & 46.4 & 84 & 100 \\
\hline
\end{tabular}

NOTE: Significant stage movements are indicated by bold type. No difference for T1 nonintenders; significant difference for T1 intenders. Arrows indicate in which direction individuals could potentially move (from nonintenders at $\mathrm{T} 1$ only forward to intenders and actors; from intenders at $\mathrm{T} 1$ backward to nonintenders and forward to actors).

group were compared separately depending on being intenders at T1 versus nonintenders at T1 (Table 1). In the matched condition (lower part of Table 1), 39 people (46.4\% of the 84 intenders receiving the intervention) progressed. Furthermore, 15 $(17.9 \%)$ regressed to nonintenders and $30(35.7 \%)$ remained in their stage. In comparison, those intenders receiving no intervention were much more likely to remain in their stage ( 21 of the 50 controls in volitional stage; $42.0 \%$ ) or to regress (16 individuals; $32.0 \%$ ) than to move forward (13 individuals; $26.0 \%$ ). Thus, in the intervention group, $20.4 \%$ more intenders progressed in the stage as compared to the control group. This difference was significant $\left(\chi^{2}=6.41, p=.02\right)$.

In the mismatched condition (upper part of Table 1$), 13$ people ( $22.4 \%$ of the 58 nonintenders in the intervention group) moved one stage forward and a further $10(17.2 \%)$ moved two stages forward (together $23 ; 39.6 \%$ of the individuals progressed) and $60.3 \%$ remained in their stage. Nonintenders in the control group were as likely $\left(\chi^{2}=0.21\right.$, $p=.45$ ) as those in the intervention group to remain in their stage (19 of the 34 nonintenders in control group; $55.9 \%$ ) or to move forward one stage ( 8 individuals; $23.5 \%$ ) or two stages ( 7 individuals; $20.6 \%$ ).

\section{Effects of the Intervention on Stage-Specific Factors (Testing Hypothesis 2)}

To test the stage-specific effects of the intervention, 2 (condition) $\times 2$ (stages) ANCOVAs of the stage-specific factors with $\mathrm{T} 1$ values as covariates were run (see Tables 2 and 3). In summary, the intervention had no significant main effects on risk awareness and outcome expectancies (expected) and planning (unexpected). We found intervention effects for intention (unexpected), self-efficacy, and behavior (both expected; see Table 3, $F$ intervention column).

Inspecting the means at T1 and T2 (Table 2), it appeared that the intention increased over time in nonintenders and decreased in intenders. Examining estimated marginal means, the intervention group reported higher intentions at T2 irrespective of stage (correspondingly, the interaction was not significant; Table 3; F Stage $\times$ Intervention 


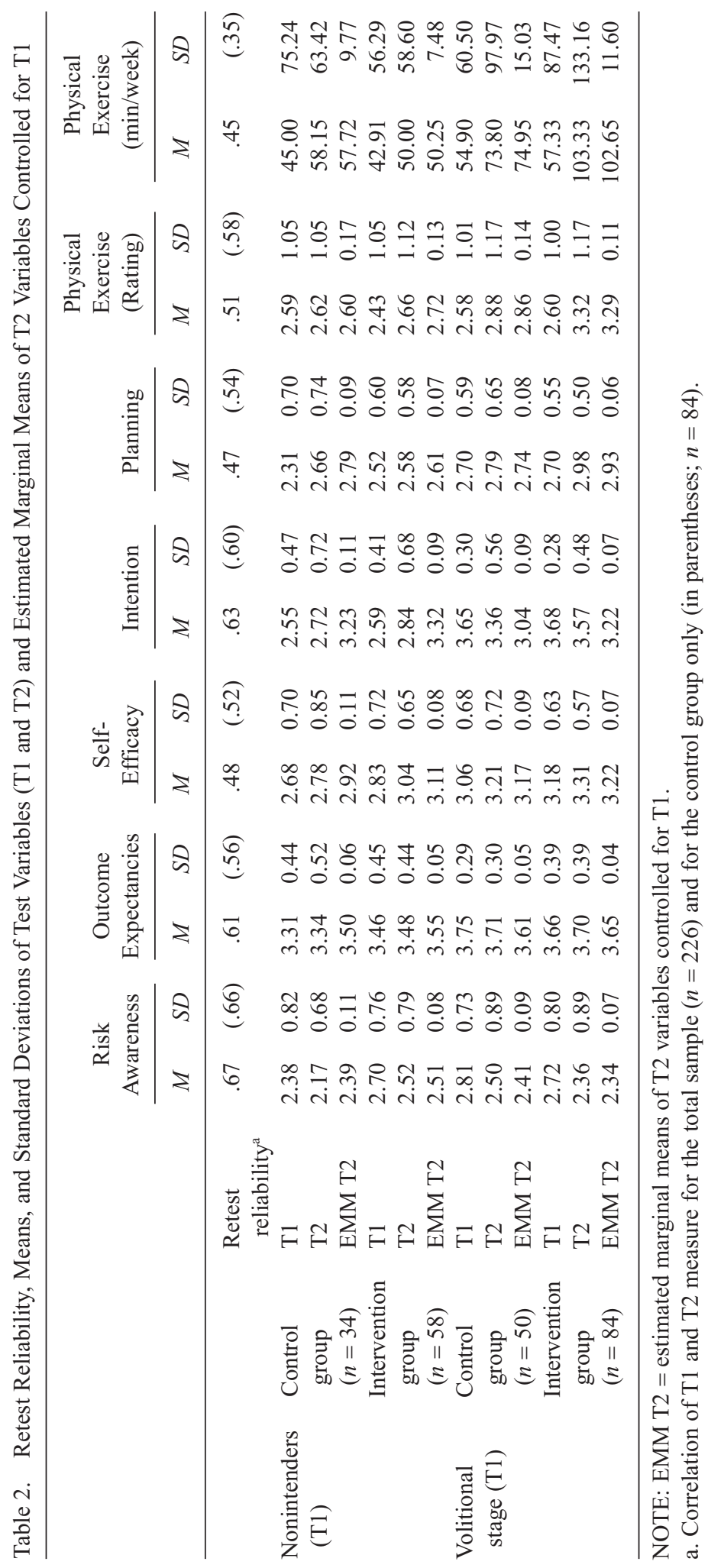




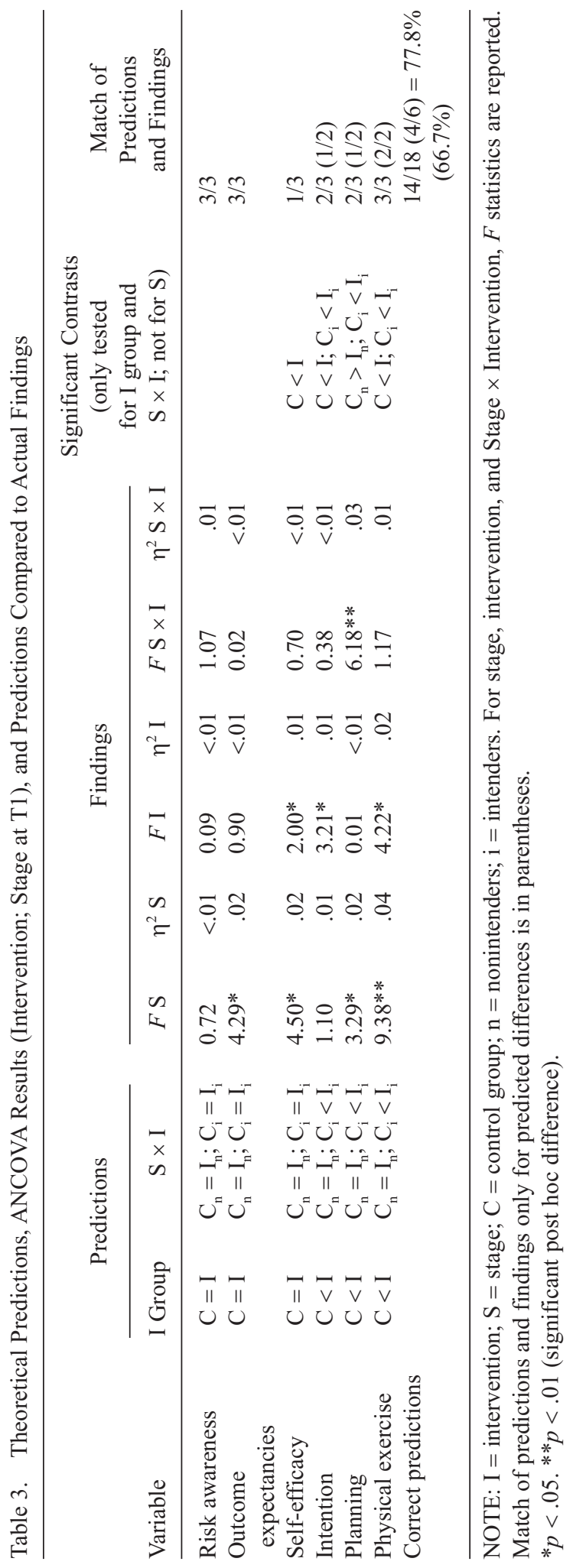


column). Self-efficacy increases were larger in the intervention group (see Table 2; No Stage $\times$ Intervention interaction, see Table 3 ). The same pattern was found for behavior: Changes in behavior were stronger in the intervention than in the control group (see Table 2; no interaction effect, see Table 3). A Stage $\times$ Intervention interaction was revealed for planning (expected, see Table 3; $F$ Stage $\times$ Intervention column). Volitional individuals in the intervention group had higher planning levels than those receiving no intervention or than nonintenders in both the control and intervention groups.

Post hoc analyses indicated that the intervention effects on intention and behavior were stronger in intenders, indicating a stage-specific effect, which was expected (Table 3). For behavior, we analyzed both the continuous measure of time and the rating scale. Results were invariant using the two different behavior measures, and the tables report the rating scale results. Of the 18 predictions depicted in Table 3, 14 were correct (77.8\%). If only predicted differences were regarded, 4 out of 6 predictions would be matched (66.7\%). The probability of finding both patterns by chance is less than 5\% (Velicer et al., 2008).

\section{Stage-Specific Mechanisms: Multiple Mediation Models (Testing Hypothesis 3)}

Finally, a multiple mediator analysis (using an SPSS macro by Preacher \& Hayes, 2008) was run to test whether the effects of the volitional intervention on behavior change could be explained by changes in intention, self-efficacy, and planning (Figure 1). These changes in stage-specific predictors and behavior were operationalized as residualized change scores obtained by regressing T2 scores on T1 scores. In a regression analysis (precondition for multiple mediator analysis), group assignment to the volitional planning intervention significantly predicted behavior change in intenders $(\beta=.43, S E=.18$, $\mathrm{CI}_{\mathrm{BCA}}=.05$ to $.43, p=.02$, expected $)$ but not in nonintenders $\left(\beta=.13, S E=.20, \mathrm{CI}_{\mathrm{BCA}}=-.16\right.$ to $.20, p=.51$, expected). Thus, subsequent analyses are reported for the volitional stage only (for coefficients for nonintenders, see Figure 1).

Group assignment predicted changes in intention $(\beta=.18, S E=.08, p=.03)$ and in planning $(\beta=.19, S E=.09, p=.04)$ but not significantly in self-efficacy $(\beta=.05, S E=$ $.10, p=.63)$. Behavior change was predicted by changes in intention $(\beta=.70, S E=.18$, $\mathrm{CI}_{\mathrm{BCA}}=.02$ to $\left..30, p<.01\right)$ and in planning $\left(\beta=.47, S E=.17, \mathrm{CI}_{\mathrm{BCA}}=.01\right.$ to $\left..25, p=.01\right)$ but not self-efficacy $\left(\beta=-.10, S E=.15, \mathrm{CI}_{\mathrm{BCA}}=-.07\right.$ to $\left..02, p=.54\right)$. After controlling for intention and planning, the relation between group assignment and behavior change was reduced to nonsignificance $(\beta=.22, S E=.17, p=.20)$. The multiple mediator model accounted for $21 \%$ of the variance (Adj. $R^{2}=.18, p<.01$ ) in behavior. Results were replicated using the continuous behavior measurements (as opposed to the Likerttype scale format; results are reported for the Likert-type scale measure of exercise) with equivalent patterns.

\section{DISCUSSION}

A randomized controlled trial tested the stage-specific effects of a stage-matched intervention. This was one of the first studies to shed light on the specific mechanisms of behavior change operating at different stages when stage-specific interventions are administered (Weinstein et al., 1998). The stage-matched intervention (volitional intervention applied to intenders) moved significantly more individuals forward in comparison to the control group (no treatment). In the stage-mismatched condition (volitional intervention for nonintenders), stage movements were not significantly 


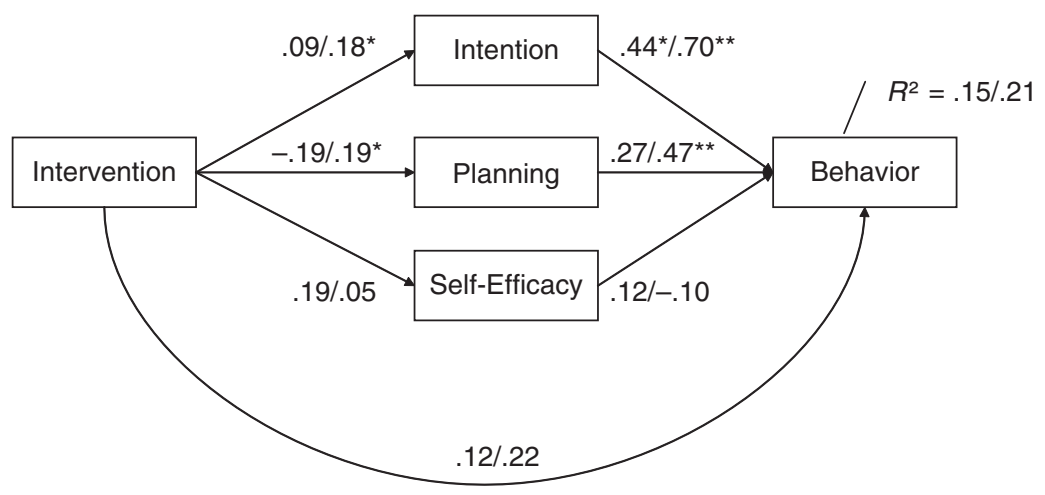

Figure 1. Multiple mediator model for the intervention effects on physical exercise. NOTE: Standardized regression coefficients for the groups of nonintenders (first coefficient) and intenders (second coefficient ).

${ }^{*} p<.05 .{ }^{* *} p<.01$.

different from those in the control group (Table 1). This finding corroborates the hypotheses and is in line with previous studies (e.g., Dijkstra, Conijn, \& De Vries, 2006).

Stage-specific effects of the intervention were found, which confirmed $77.8 \%$ of our hypotheses (see Tables 2 and 3). If only predicted differences and no predicted similarities were regarded, this match would be reduced to $66.7 \%$ (see Table 3). Still, this is quite a good match of hypotheses and empirical findings (Velicer et al., 2008) that supports the stage-specific view on relevant variables at the particular stages. As this was the first study testing stage-specific effects with this strategy, it is difficult to compare the current findings to previous study results. The analytical procedure can rather be seen as support for stage assumptions and possible means to test these in more detail in the future.

The finding that $17.9 \%$ of the participants in the matched condition (intenders, volitional intervention) unexpectedly regressed to nonintenders might be related to the imperfect validity of the stage measure (Lippke et al., 2009), which might have misclassified these specific participants as being in the volitional stage while actually being nonintenders, thus receiving an intervention mismatched to their stage-specific needs. Previous research has demonstrated that receiving a volitional intervention while being a nonintender might have negative effects on both behavior (Lippke et al., 2004) and study retention (Schüz, Wiedemann, Mallach, \& Scholz, 2009). These detrimental effects might be because of "demotivation" effects (Glasgow \& Orleans, 1997) if unmotivated participants are forced to elaborate on behavior that is of no importance to them.

Finally, multiple mediator models revealed stage-specific mediation mechanisms (Figure 1). In intenders, intention and planning mediated the effects of the matched intervention on behavior change (no effect in the mismatched condition, i.e., nonintenders). That is, multiple mediator analyses confirmed the main expected stage-specific mechanisms. However, the hypothesis regarding the mediating role of self-efficacy was not supported. This might be because of the fact that the volitional intervention did not explicitly target self-efficacy. In further volitional interventions, self-efficacy could be promoted with model learning and verbal persuasion. A reason why the volitional intervention affected intention in the present study might be that it also helped to keep the intention high. 
The rather small effects should be appreciated with the consideration that the treatment was a rather low-key and simple Web-based intervention. In comparison to typical intervention studies, the treatment in this study was much shorter and less time-consuming (Adams \& White, 2008; Bridle et al., 2005; Craig et al., 2003; Dijkstra et al., 2006). The $21 \%$ variance accounted for by the intervention in intenders is comparable even to correlational HAPA studies (e.g., Schwarzer et al., 2007-21\%; Schwarzer et al., 2008$14 \%$ to $39 \%$ ). Thus, even if the range of variance is limited because of splitting the total sample into stage subgroups, a similar amount of variance can be explained by the relevant predictors. Even if less than one fourth of the variance is explained, the study offers important insights into the behavior change processes of the subsample of volitional but yet inactive individuals: Here, the effects of a volitional intervention are mediated by intention and planning.

Some implications for future research need to be mentioned. The current data are based on online self-reports. Online studies give researchers the potential to reach large samples of people with diverse socioeconomic statuses and ages and from different geographic regions (Rademacher \& Lippke, 2007) and have proven to be as scientifically sound as data collected via traditional methods (Gosling, Vazire, Srivastava, \& John, 2004). Our data are not representative for the German general population, as our study sample overrepresented women and individuals with higher education. It appeared, however, roughly representative in comparison to other online studies (see Wiedemann et al., 2009). Moreover, as the primary aim of this study is to provide evidence on the feasibility and practicability of implementing stage-specific interventions, the validation of these interventions in a general population is a challenge for further studies. Furthermore, the differentiation of our sample according to a between-individuals factor (stage) implies that the main target population of such interventions is to be identified according to psychological and behavioral criteria such as stage rather than populationbased criteria to be maximally effective. Future studies might want to replicate our findings with samples that are recruited in a more proactive manner (e.g., in a workplace setting, in schools, or in hospitals; see Prochaska et al., 2008). Although the validity of self-reports on physical exercise appears to be satisfactory and the utilized assessment was previously validated, further research might want to replicate the results with objective measures (e.g., using pedometers or accelerometers). However one has to bear in mind that both self-report and objective data have limitations and tap different facets of the phenomenon being measured (Prince et al., 2008). Furthermore, only short-term effects were investigated. Long-term effects should be studied in more depth in the future (Adams \& White, 2008). Although replications are always desirable, generalizability of the present findings is supported by evidence from a representative online sample and from theory-based analyses.

\section{Conclusions}

The findings from this study, namely, the identification of planning and intention as stage-specific mediators of a stage-specific behavior change intervention, help to explain the process of behavior change and thus shed more light on the "black box" of what actually happens in interventions. In the future, studies should follow this logic and thus contribute to a growing evidence and knowledge base on effective behavior change processes.

Saving resources by optimizing the efficacy of interventions can be accomplished by first assessing the stage (whether or not individuals intend to adopt a health behavior) 
and then applying only the matching intervention: If individuals have already formed the intention to adopt a new behavior, then planning should be facilitated. The success of the intervention should be evaluated with stage-relevant variables: If a volitional intervention were applied, then only volitional variables would indicate success of the intervention (beside behavioral outcomes and stage progression).

\section{Implications for Practice}

Assessing a person's stage facilitates matching interventions: Individuals who are highly motivated benefit from planning (when, where, how, and what to do in the face of obstacles) the behavior they intend to adopt. On the contrary, individuals who are not motivated to change need an intervention to become motivated. Also, if stages are being assessed, then one can take advantage of this construct to evaluate putative stage-specific intervention effects: To expect that nonintenders would immediately adopt a new behavior is not realistic, as they first need to form an intention before the volitional intervention helps to translate this intention into actual behavior change. Stage movements (e.g., deciding to change, preparing for actual changes) should be regarded as outcomes: Moving participants from one stage to the subsequent one can be seen as an intervention success, even if actual behavior does not yet materialize. In interventions, such stepwise changes can be easily fed back to the program participants and individuals can be further motivated to keep on going.

\section{References}

Adams, J., \& White, M. (2008). Health behaviours in people who respond to a Web-based survey advertised on regional news media. European Journal of Public Health, 18(3), 335-338.

Bridle, C., Riemsma, R. P., Pattenden, J., Sowden, A. J., Mather, L., Watt, I. S., et al. (2005). Systematic review of the effectiveness of health behavior interventions based on the transtheoretical model. Psychology \& Health, 20(3), 283-301.

Craig, C. L., Marshall, A. L., Sjoestroem, M., Bauman, A. E., Booth, M. L., Ainsworth, B. E., et al. (2003). International Physical Activity Questionnaire: 12-country reliability and validity. Medicine and Science in Sports and Exercise, 35(8), 1381-1395.

Dijkstra, A., Conijn, B., \& De Vries, H. (2006). A match-mismatch test of a stage model of behaviour change in tobacco smoking. Addiction, 101(7), 1035-1043.

Enders, C. K. (2001). A primer on maximum likelihood algorithms available for use with missing data. Structural Equation Modeling, 8(1), 128-141.

Glasgow, R. E., \& Orleans, C. T. (1997). Adherence to smoking cessation regimens. In D. S. Gochman (Ed.), Handbook of health behavior research II: Provider determinants (pp. 353-377). New York: Plenum.

Gollwitzer, P. M., \& Sheeran, P. (2006). Implementation intentions and goal achievement: A metaanalysis of effects and processes. Advances in Experimental Social Psychology, 38, 69-119.

Gosling, S. D., Vazire, S., Srivastava, S., \& John, O. P. (2004). Should we trust Web-based studies? A comparative analysis of six preconceptions about Internet questionnaires. American Psychologist, 59, 93-104.

Lippke, S., Ziegelmann, J. P., \& Schwarzer, R. (2004). Initiation and maintenance of physical exercise: Stage-specific effects of a planning intervention. Research in Sports Medicine, 12, 221-240.

Lippke, S., Ziegelmann, J. P., Schwarzer, R., \& Velicer, W. F. (2009). Validity of stage assessment in the adoption and maintenance of physical activity and fruit and vegetable consumption. Health Psychology, 28(2), 183-193. 
MacKinnon, D. P., Lockwood, C. M., \& Williams, J. (2004). Confidence limits for the indirect effect: Distribution of the product and resampling methods. Multivariate Behavioral Research, 39, 99-128.

Preacher, K. J., \& Hayes, A. F. (2008). Asymptotic and resampling strategies for assessing and comparing indirect effects in multiple mediator models. Behavior Research Methods, 40(3), 879-891.

Prince, S. A., Adamo, K. B., Hamel, M. E., Hardt, J., Gorber, S. C., \& Tremblay, M. (2008). A comparison of direct versus self-report measures for assessing physical activity in adults: A systematic review. International Journal of Behavioral Nutrition and Physical Activity, 5, 56-79.

Prochaska, J. O., Wright, J. A., \& Velicer, W. F. (2008). Evaluating theories of health behavior change: A hierarchy of criteria applied to the transtheoretical model. Applied Psychology: An International Review, 57, 561-588.

Rademacher, J. D. M., \& Lippke, S. (2007). Dynamic online surveys and experiments with the free open-source software dynQuest. Behavior Research Methods, 39(3), 415-426.

Scholz, U., Schüz, B., Ziegelmann, J. P., Lippke, S., \& Schwarzer, R. (2008). Beyond behavioral intentions: Planning mediates between intentions and physical activity. British Journal of Health Psychology, 13, 479-494.

Scholz, U., Sniehotta, F. F., \& Schwarzer, R. (2005). Predicting physical exercise in cardiac rehabilitation: The role of phase-specific self-efficacy beliefs. Journal of Sport \& Exercise Psychology, 27(2), 135-151.

Schüz, B., Wiedemann, A. U., Mallach, N., \& Scholz, U. (2009). Effects of a brief behavioural intervention for dental flossing: Randomized-controlled trial on planning when, where and how. Journal of Clinical Periodontology, 36, 498-505.

Schwarzer, R., Luszczynska, A., Ziegelmann, J. P., Scholz, U., \& Lippke, S. (2008). Socialcognitive predictors of physical exercise adherence: Three longitudinal studies in rehabilitation. Health Psychology, 27(1), S54-S63.

Schwarzer, R., Schüz, B., Ziegelmann, J. P., Lippke, S., Luszczynska, A., \& Scholz, U. (2007). Adoption and maintenance of four health behaviors: Theory-guided longitudinal studies on dental flossing, seat belt use, dietary behavior, and physical activity. Annals of Behavioral Medicine, 33(2), 156-166.

Velicer, W. F., Cumming, G., Fava, J. L., Rossi, J. S., Prochaska, J. O., \& Johnson, J. (2008). Theory testing using quantitative predictions of effect size. Applied Psychology: An International Review, 57(4), 589-608.

Weinstein, N. D., Rothman, A. J., \& Sutton, S. R. (1998). Stage theories of health behavior: Conceptual and methodological issues. Health Psychology, 17(3), 290-299.

Wiedemann, A. U., Lippke, S., Reuter, T., Schüz, B., Ziegelmann, J. P., \& Schwarzer, R. (2009). Prediction of stage transitions in fruit and vegetable intake. Health Education Research, 24, 596-607.

Ziegelmann, J. P., Lippke, S., \& Schwarzer, R. (2006). Adoption and maintenance of physical activity: Planning interventions in young, middle-aged, and older adults. Psychology \& Health, 21(2), 145-163. 